International Journal of Child, Youth and Family Studies (2018) 9(1): 54-68

DOI: http://dx.doi.org/10.18357/ijcyfs91201818119

\title{
CREATING EVIDENCE-BASED CHANGE THROUGH A TRAUMA- INFORMED LENS: TRANSLATING PRINCIPLES INTO PRACTICE
}

\author{
Susan Hunt, Marlene M. Moretti, Chris Booth, and Nickole Reyda
}

\begin{abstract}
When programs and services incorporate an understanding of trauma and its impact on an individual's behaviour and ability to cope, the potential for misdiagnosis and inadequate treatment planning is significantly reduced. Incorporating trauma-informed approaches into service delivery is an essential component to developing programs that accurately address the needs of youth and their families. The organization involved in this study, in the Province of British Columbia, Canada, provides an extensive array of services to youths aged 12 to 18 years who have significant emotional, behavioural, and psychiatric difficulties. In a joint multidisciplinary effort to better support traumatized young people and their families, the organization embarked on an in-depth evaluation of its service delivery. Together the team co-created a shift in practice that supported the translation of trauma-informed principles into practice and developed valid and measurable methods for evaluation through the adoption of a participatory action framework. Four semi-structured interviews were developed for collecting qualitative feedback from clients, stakeholders, and staff who experienced the change in service delivery across 5 clinical cases over the course of 8 months. The feedback confirmed that the shift in practice was effective in cultivating an environment of safety, choice, and collaboration for clients. This resulted in the development of an evidence-based shift in service delivery as well as identifying training needs and developing plans to integrate this change into broader practice throughout the organization.
\end{abstract}

Keywords: trauma informed, principles, qualitative research, Maples Adolescent Treatment Centre, the Maples, evidenced-based, service delivery

Susan Hunt BA (corresponding author) is a Certified Child and Youth Care Professional with the Ministry for Children and Family Development, Bldg \#200; Suite \#208- $2043464^{\text {th }}$ Ave, Langley, British Columbia V2Y 1N4. Email: Susan.M.Hunt@gov.bc.ca

Marlene M. Moretti PhD is a Tier 1 Canadian Research Chair and Professor in the Department of Psychology at Simon Fraser University, Burnaby, British Columbia V5A 1 S6. Email: moretti@sfu.ca

Chris Booth MD is a Child and Adolescent Psychiatrist and Clinical Director of the Maples Adolescent Treatment Centre, Burnaby, British Columbia V5G 3H4. Email: $\underline{\text { Chris.Booth@gov.bc.ca }}$

Nickole Reyda is a Registered Psychiatric Nurse and Program Coordinator at the Maples Adolescent Treatment Centre, Burnaby, British Columbia V5G 3H4. Email: Nickole.Reyda@gov.bc.ca 
International Journal of Child, Youth and Family Studies (2018) 9(1): 54-68

When people think of the term "trauma", they generally think of things like a catastrophic world event or a devastating personal tragedy. However, to those in the helping professions, it is recognized that trauma is a much more common occurrence with far reaching and pervasive consequences on a person's life and ability to cope. In fact, according to British Columbia's Trauma-Informed Practice Guide (BC Provincial Mental Health and Substance Use Planning Council [BCMHSUPC], 2013), a remarkable " $76 \%$ of Canadian adults report some form of trauma exposure in their lifetime" (p. 9).

There are a number of dimensions of trauma that take into account the magnitude of an incident, the frequency of its occurrence, and its duration, as well as if it occurs within an interpersonal relationship or from an external source. Whether it is a single life event, a subtle series of attachment injuries and adverse childhood experiences, or a prolonged exposure to abuse, trauma may result in devastating physical and psychological consequences. Furthermore, while reactions to trauma can vary from person to person, the age at which trauma occurs is an important variable (BCMHSUPC, 2013). When trauma is experienced in early childhood, it can have a significant impact on the development of the brain, the progression of social and emotional growth, and a person's behaviour (Anda et al., 2006). In fact, according to the Adverse Childhood Experiences (ACE) study by Anda and colleagues (2006), adverse childhood experiences are much more common than previously recognized and have been directly linked to substance use and mental health problems later in life. It is therefore crucial to integrate trauma-informed approaches into service delivery as an essential component in creating and developing programs that best fit the needs of clients. In fact, when services do not reflect an understanding of trauma and its impact on a person's attempts to cope, the potential for misdiagnosis and inadequate treatment planning can be substantial (BCMHSUPC, 2013).

Even when the importance of trauma-informed practice is recognized, transforming agency practices to align with a trauma-informed approach can present a number of challenges. This is particularly true for multidisciplinary agencies that provide a variety of programs to youth and families, including both on-site and community-based services. The organization involved in this study, the Maples Adolescent Treatment Centre (the Maples; BC Ministry of Children and Family Development, n.d.), has been in operation since 1969 and is part of the Provincial Child and Youth Mental Health service network for British Columbia. The Maples provides an extensive array of programs and services to youth aged 12 to 18 throughout the province who have significant emotional, behavioural, and psychiatric difficulties; the clients include youth who have experienced significant trauma both individually and multi-generationally. A high proportion of clients receiving service are youth from economically disadvantaged families, youth within the foster care system, and Aboriginal families. While the organization utilizes the language and diagnostic criteria of the Diagnostic and Statistical Manual for Psychiatric Disorders (DSM), the foundations and principles of the existing clinical model are explicitly based on the recognition of attachment as a fundamental biological regulatory system that shapes behaviour. Because attachment-based practice inherently recognizes the impact of trauma on mental health and 
International Journal of Child, Youth and Family Studies (2018) 9(1): 54-68

behaviour, clinical practice that arises from an attachment-based perspective aligns well with emerging trauma-based principles.

In recognition that the overarching goal was to establish quality services that are both evidence-based and trauma-informed, the Maples endeavoured to ensure that the programs delivered not only promote child and adolescent well-being, but also provide services that do not retraumatize families.

\section{Project Objective}

To facilitate a review of clinical practice in relation to Trauma-Informed Practice guidelines, a collaborative practice team (CPT) comprising a multidisciplinary working group and a steering committee was created at the Maples in 2014. Within this initiative, the organization embarked on an in-depth evaluation of service delivery that endeavoured to deepen understanding of the relationship between trauma and a person's attachment needs.

A participatory action model was adopted to guide this work; it incorporates strengthening collaborative engagement, ongoing feedback, and learning throughout the process (Bergold \& Thomas, 2012). This approach ensured the collection of meaningful information about the quality and effectiveness of services while also creating a collaborative context that supported clinically relevant, feasible, and reliable changes in service delivery. The qualitative approach used in this study, which was designed to provide structure and accountability to a project and to clarify the purpose and direction of the research, provided a clear framework to guide the various methods of data collection, the analyses, and the interpretation of the findings into evidence (Bouma, Ling, \& Wilkinson, 2012).

Using procedures already established within the organization, the delivery of programs and services within the Maples is developed and evaluated in partnership with youth, caregivers, and community care teams, and through research, education, and collaboration (Maples Adolescent Treatment Centre, 2014). Based on the feedback from the organization's outcome measures, and from discussions within the CPT, several key areas were identified for practice review and key questions were posed:

- Is the organization's service delivery consistent with best-practice guidelines and evidenced-based practice in the field of child and youth mental health?

- How might we integrate the principles of a trauma-informed approach into the areas of service delivery and program development?

Together, staff identified the care plan development meeting and its preceding assessment process as the key focus for evaluation (see Figures $1 \& 2$ ). 
International Journal of Child, Youth and Family Studies (2018) 9(1): 54-68

\section{The Care Plan}

A care plan at the Maples is a comprehensive assessment that includes four components:

- A child and youth care/psychiatric nursing report from a life space perspective

- A social history report of the family's generational themes and patterns, completed by a social worker

- A psychological assessment that includes formal cognitive and psychological testing as well as an interview with the youth

- An educational assessment that includes academic testing and a review of school records

At the end of this 4-week process, each of the above disciplines presents their findings in a care plan development meeting at which the family is in attendance, along with community professionals and the assigned Maples psychiatrist. The care plan document, developed from the information shared in this meeting, is written by the psychiatrist and is designed to be a "portable" document: the information and recommendations formulated are intended to follow the youth throughout their adolescence and into any community or setting in which the youth lives. A care plan consultant is then assigned to this document and it is this person's role to assist others in understanding and adapting the recommendations from the care plan to work with the resources available within the youth and family's community.

Figure 1. Development of a care plan at the Maples prior to review.

Together the CPT developed a shift in practice regarding the care plan development meeting that it hypothesized would not only be effective in responding to the concerns and feedback from the organization's outcome measures, but would also support the translation of trauma-informed principles (TIPs) into practice. This led to the creation of a formulation meeting for the Maples team that would be scheduled to occur prior to the care plan development meeting itself. This pre-meeting was seen as an opportunity for the care plan team to discuss detailed results from the multidisciplinary evaluations, to identify common themes across the assessments, and to ensure that the information shared in the care plan development meeting was presented in a traumasensitive format that focused on patterns, themes, and strengths relevant to the youth and family. The formulation meeting was also viewed as an opportunity to shape the presentations in the care plan development meeting so that they could be tailored to the needs of clients rather than the other professionals. 


\section{Youth and Family Intake}

- Youth and family attend a meeting where the care plan process is explained

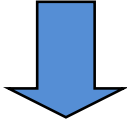

\section{Multidisciplinary Assessment}

- Assessments completed by Psychiatry, Social Work, Psychology, Education and Youth Care

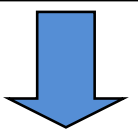

Care Plan Meeting with Youth, Family and Community Support

- Professionals present assessment findings

- Care plan is discussed as an integrative document that brings together observations and recommends treatment options and community supports

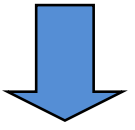

\section{Discharge to Community}

- Outreach workers are introduced and provide follow up support to community to implement the care plan

Figure 2. Complete care plan process prior to review.

\section{Method}

Bouma et al. (2012) stated that a guiding principle of doing research is letting the research question itself determine the data collection strategy. Given that our research questions were aimed at determining the impression left by, and the emotive experience resulting from, a change in service delivery, we utilised qualitative methods for our research. This research project had an element of "live-learning": the focus was not a formal evaluation of an already established and standard practice but rather an evaluation of a process of change that was current and ongoing. A 
International Journal of Child, Youth and Family Studies (2018) 9(1): 54-68

qualitative approach was needed to provide us with the ability to adapt and modify the interviews and the variables as required by the participant feedback and themes that emerged besides the four TIPs used to guide this review (see Figure 3).

\section{Trauma-Informed Principles}

Trauma Awareness: Building awareness and understanding for clients and staff around the impact of trauma and the relationship between trauma and substance use, physical health, and mental health.

Safety and Trust: Establishing physical, emotional, and cultural safety for both clients and staff. Safety and trustworthiness can be established through adapting physical spaces, providing clear information, ensuring consent and collaboration, and so on.

Choice, Connection, and Collaboration: Fostering a sense of self-determination, efficacy, dignity, and personal control for those receiving service by providing choice and working collaboratively with clients.

Strength and Skill Building: Assisting clients in identifying strengths to further develop resiliency and coping skills.

(BCMHSUPC, 2013)

Figure 3. The four TIPs used to guide this review.

\section{Sample}

A smaller pilot team from within the larger CPT, comprising a professional from each of the discipline areas involved in the care plan assessment, was assembled to implement the new format of the care plan development meetings. Five upcoming clinical cases were identified as proposed pilot cases for the new format. For the purposes of this study, and because the Maples is an organization that serves the entire province of British Columbia, the pilot cases were selected based on geographical location. Selection by proximity and accessibility to the youth, family, and community professionals allowed the research team to collect the qualitative feedback for evaluating the impact of the shift in service. The five clinical cases were chosen to represent diversity in family composition, culture, and socioeconomic status.

\section{In-Depth Interview}

While the larger CPT worked together in defining their roles and functions within the formulation meeting versus the care plan development meeting, the interdisciplinary steering committee began the work of developing valid and measurable methods for evaluation. A high priority was placed on obtaining feedback from those who directly experienced the new format as 
International Journal of Child, Youth and Family Studies (2018) 9(1): 54-68

these contributions were recognized as essential for measuring the effectiveness of developing a stronger and more trauma-informed approach. Therefore, as guided by the TIPs outlined in Figure 3 , four semi-structured interviews were developed for collecting qualitative feedback from those who had experienced the shift in practice: clients, stakeholders, and the Maples professionals.

Adopting the TIPs into the development of these interviews provided a framework for creating research methods designed to measure and examine the translation of the principles into practice. Questions were carefully considered for each of the TIP categories and were aimed towards evaluating whether the shift in practice demonstrated trauma awareness, promoted an environment of safety and trust for clients, and provided a sense of collaboration, choice, and connection for the youth, family, and community care team. Questions were also aimed at determining the level to which the youth's and family's strengths were identified. Additionally, several questions were created for measuring the level of collaboration, teamwork, and support amongst the Maples team, with the intent of addressing the evidence of vicarious trauma experienced by professionals.

\section{Field Research}

In addition to the in-depth interviews, we decided bring in an element of field research a silent observer - for the aggregation of qualitative data. A member from the child and youth care discipline within the Maples CPT was designated the silent observer, a neutral, nonparticipating attendee in each of the pilot case care plan meetings who would record the events, behaviours, and dynamics that occurred within each meeting. This data provided supplementary evidence to the feedback collected from the in-depth interviews.

With the exception of the final pilot case care plan meeting for which a CPT member from the psychiatric discipline filled in, the designated professional in the silent observer role did not vary throughout the project.

\section{Inter-rater Consensus}

During the progression of data collection and theming of the qualitative feedback gathered from each of the pilot cases as they occurred, the feedback was also presented to the members of the larger CPT. Not only did this process reaffirm the collaborative and participatory action framework adopted throughout this research process, but it also provided an opportunity to assess consensus in the theming of the qualitative feedback and created cross-rater reliability in the interpretation of the data.

\section{Limitations}

In a large organization such as the Maples, the adoption of a trauma-informed perspective requires collaboration across disciplines to ensure a coherent framework is created that guides services within each area of specialization. Well established institutions with a long standing history and model of service delivery also face the challenge of reviewing administrative and clinical practices and programs that may define their identity as an organization. Retaining aspects 
International Journal of Child, Youth and Family Studies (2018) 9(1): 54-68

of practice that are congruent with trauma-informed practice while at the same time introducing change where it is needed can be challenging. It requires a collaborative approach that engages staff at all levels and is guided by a clearly defined framework and timeline.

A guided research approach using the qualitative model and methods for evaluation provided ongoing opportunity to shape and refine problems as they arose throughout the research project. Some of the problems and difficulties that arose involved three occurrences when the family or community professionals declined consent to have their interviews audiotaped. This made transcribing their interviews more difficult, as the interviewer needed to take notes while conducting the interview. Not only did this hamper the ability of the interviewer to be fully present in gathering the authentic experience of these participants, but it also affected the accuracy of the transcriptions.

Furthermore, there were other difficulties: two instances of delayed care plans due to client circumstances, a parent and youth who withdrew their consent to participate in the interview following their care plan meeting, a community professional who was unavailable to participate in her interview due to illness, and a few occurrences of having to adapt and complete the interviews over the telephone because of hesitance on the part of a youth, a caregiver, and several community professionals in committing to an in-person interview.

Finally, as the approach to this project primarily engaged those professionals who were most interested in the process of change and open to creating a shift in practice, the selection of the pilot team participants was later recognized as one of the limitations of the research.

\section{Findings}

Adopting the TIPs to guide this project not only provided clear structure in the development of the qualitative methods for evaluation; they also guided the analysis and interpretation of both the feedback gathered and the observational data obtained by the silent observer.

\section{Trauma Awareness}

Throughout all five pilot cases, clients as well as stakeholders were consistent in their responses that the information presented in the meeting was helpful to them in understanding how past trauma and events were related to current challenges the family was experiencing. Both groups found that the meeting was effective in "helping us to understand [the youth] more"; that it "helped make meaning of [the youth's] behaviour"; and that it provided them with a "sense of how to approach working with [the youth]". One parent in Pilot Case \#1 responded that the meeting and the way in which the information was shared helped her in answering "the whys" behind her child's behaviour.

The observational data substantiated the feedback; when the reports were presented in a summary focusing on themes and patterns, the families appeared more engaged and the youth were 
International Journal of Child, Youth and Family Studies (2018) 9(1): 54-68

able to remain in the room for the entire meeting. Conversely, when there appeared to be some practice drift from the project's objective in Pilot Cases \#3 and \#4, the opposite observations were made. For example, during these care plans, the staff presented their reports in full detail. This practice demonstrated decreased awareness regarding the impact of trauma and retraumatization; the silent observer noted that the families tended to present as more distracted with "glazed over" expressions during these meetings. During the meeting for Pilot Case \#4, the youth left the room until the presentation of the family's social history report was over.

In terms of the feedback from the Maples team, there was consistency in reporting that the formulation meeting was effective in providing a shared understanding of the family and community dynamics prior to entering the care plan meeting. It was further reported that this resulted in the team's ability to adapt the process within the care plan meeting itself to meet the youth's and family's needs more effectively. For example, in several of the formulation meetings, the team was able to change the order in which the reports would be presented in the care plan meeting based on what the youth's strengths were and what reports the team thought might contain more difficult information. As well, the team reported that this new format helped them in "simplifying who the audience is" and prepared them to "better attune to the family's needs and reactions".

\section{Safety and Trust}

Across all five pilot cases, the families as well as community professionals were consistent in reporting that the environments within the meetings were "very sensitive and respectful" and that the families were "treated very well" owing to the approach of the care plan team. Furthermore, community stakeholders felt better about recommending the service due to the environment of safety that was created within the new format, saying, for instance: "I would have struggled with feeling protective of the family previously." and, "Now I feel like with more sensitivity the likelihood of it being a difficult process is less".

The feedback from caregivers was also consistent with regard to an overall sense of safety in the meetings. For example, $100 \%$ of caregivers responded "no" to the question: "Did you feel unsafe or exposed by the information shared in the meeting?" and several parents also reported feeling respected when they expressed their concerns that sensitive information from their social history reports would be read out in the meeting. In fact, in one of the pilot cases, a caregiver reported specifically that "I had asked for some stuff to be left out (during the meeting) and I was pleased that it was respected".

The observational data provided indicate that the discussions within the formulation meetings were also effective for the team in creating an environment of safety and trust for families. By framing their role as a collaborative partnership with the family and community professionals, and mindfully seating themselves in the meeting room to be intermixed with the group, power differentials in the room were minimized (BCMHSUPC, 2013). This perspective 
International Journal of Child, Youth and Family Studies (2018) 9(1): 54-68

and mindful seating arrangement was also effective in providing a sense of availability, support, and proximity to the clients. For example; the silent observer noted:

As [Maples social worker] had close physical proximity to mom, she was able to note her discomfort with portions of the report and touched her arm; offering her comfort at a point in the report as well. [Mom was] offered Kleenex.

As [Maples social worker] summarized the report, it gave her opportunity to make eye contact with mom and [youth]. Mom was engrossed in reading along with the report and didn't notice the attempt for eye contact, but [youth] did meet [social worker's] eyes.

Feedback from the staff also revealed a consensus that the formulation meeting provided a venue for discussion and planning of any necessary adaptations to the structure of the care plan meeting and for considering any dynamics and relationships amongst the participants who would be attending. For example, as was discussed above under Trauma Awareness, in three of the five pilot cases the team decided to switch the order in which reports would be presented in order to cultivate an immediate environment of safety for clients. While traditionally the family's Social History is the first report presented in a care plan meeting, in these three pilot cases, the team chose to begin with the report that best represented the strengths of the youth and family. Additionally, with specific reference to two of the pilot cases that involved families with Aboriginal heritage the team reported that the formulation meeting provided an important venue and context for reflecting on cultural issues related to historical and continuing cultural marginalization, and to increase their awareness and understanding of transgenerational and systemic trauma experienced by these families. These discussions were essential to the team's ability to provide cultural safety for these families as well as respect for their heritage and experiences.

\section{Choice, Collaboration, and Connection}

Questions within the semi-structured interviews were designed to seek feedback in two aspects of this TIP category: (a) the availability of choice to the clients and their sense of collaboration and connection within the meeting; and, (b) the sense of connection and collaboration within the Maples care plan team.

Throughout the five pilot cases, feedback was $100 \%$ consistent across families, community teams, and Maples staff that families had the choice of whether to participate or not, and that the new format of the care plan meeting was effective in promoting an environment of choice and collaboration for clients. Common feedback included: "The biggest difference is that there is a lot more dialogue with the family" (stakeholder); "There was so much participation, it was very respectful" (stakeholder); "The [youth] was engaged and it felt like we did it with her, not to her" (Maples staff); and, "I really enjoyed being a part of this care plan" (stakeholder). Families also reported that the opportunity for choice and collaboration resulted in feelings of control and added 
International Journal of Child, Youth and Family Studies (2018) 9(1): 54-68

a sense of safety for them within the meeting. In fact, one parent responded, "The social worker was respectful of my choice so I felt like I had control."

As mentioned previously, not only did this new model provide an opportunity to increase the level of choice, collaboration, and connection for clients and stakeholders, but the Maples professionals also reported an increased sense of collaboration and connection amongst themselves as a team. Staff comments included descriptions of the formulation meeting as "remarkably casual and there was good dialogue amongst everyone"; "quite a harmonious sort of meeting"; "there was a real sense of 'team' as we went into the meeting"; and, "I was validated and supported by my team". Furthermore, it was acknowledged with $100 \%$ consistency in all staff responses that this format was "better" than the previous model and that the formulation meeting was effective in eliminating the need to track each other down and consult during the lead-up to a care plan development meeting.

\section{Strengths and Skill Building}

Across all five pilot cases there was consensus throughout the feedback that the new model was effective in producing a greater focus on the youth's and family's strengths and protective factors. It was further reported that this stronger focus generated greater opportunities for skill building and effective treatment planning on the part of the community professionals working with the youth and family. For example, one community mental health clinician reported that the focus on strengths "helped us and the family find a fresh start to work from".

Further responses from community professionals included: "Everyone really emphasised [youth's] strengths"; and, "The reports were very respectful and really built up the strengths of [parent]". Reflecting on the difference between prior practice and the revised practice format, another community professional commented: "Before it was about the diagnosis and this time it was about identifying ways to support the youth so strengths were the focus."

Most importantly, when asked if they felt their strengths were recognized within the meeting, $100 \%$ of clients responded "yes". Despite feedback from one youth that it "was a bit awkward", there was strong consensus in the youth and family responses that they felt "good" and "hopeful" after their care plan meetings.

\section{Practice Reflection}

Even though the feedback across the TIP domains from families, community professionals, and staff was almost universally positive, several areas of practice emerged as needing further development and growth. First, staff consistently expressed a need for greater focus and structure

within the formulation meeting, including feedback such as: "The formulation meeting is really unstructured"; "I really want it to be chaired and everyone take turns"; and, "It should be chaired better so there is no competing for time", with the further comment, "No, I did not feel heard or respected". Furthermore, when specifically asked what they might change about the formulation meeting in the future, staff responses were consistent: "People need to have an awareness of 
timelines and focusing on themes"; "We could focus the discussion better and tighten the timeframe"; "I think it was hard for [a staff member] because they didn't come prepared to discuss themes, they used the formulation meeting for this purpose"; and, "People need to come prepared with their themes and TIPs in mind already because there is not a lot of time". These comments are especially important when considered alongside comments already noted on the value of the formulation meeting in creating a shared understanding of trauma issues for the families and support for staff in processing trauma information. The need for clarity, structure, and safety in the formulation meeting was clearly just as important as the need for these same aspects in the care plan meeting. In fact, as the team progressed they realized that the TIP principles applied as much to them as to the families that they served, and that integrating these principles into their team relationships and process was essential in supporting their emotional and professional responsiveness to their clients.

A second recurring topic, related to practice reflection across the pilot cases, was how to effect a sense of closure at the end of a care plan meeting and the importance of a relational continuity. Staff agreed that greater attention was needed to ensuring that families in the assessment process were served by the same people as much as possible. For example, prior practice allowed for and sometimes required that professionals have someone else stand in for them to present their findings at a care plan meeting due to scheduling conflicts. Staff consistently pointed to their discomfort with this process as such disruption could easily be experienced as abandonment or disregard by clients who have experienced many such disruptions in their lives. The team and the agency struggled to find ways to balance workload and scheduling problems with their commitment to the TIP principles and the welfare of their clients. All agreed that further discussion of and attention to systemic and institutional issues were needed.

Staff also expressed concern about the opening and closing of the care plan assessment and the importance of ensuring respect and structure throughout this process. Through practice reflection, the team recognized the importance of having the care plan consultant, who is responsible for the post-discharge community support, involved right from the start of the admission process. Given the care plan consultant's continued role with the family, providing continuity across service delivery represented an important commitment and point of contact for families. Discussions within the larger CPT, as well as feedback from the qualitative interviews, indicated an ongoing "breakdown in the flow of the meeting" at its end when attention shifted to the care plan consultant explaining his or her ongoing role to the family and community team. For example, one care plan consultant commented:

What I am struggling with now is how to close the meeting. Before I would invite the Maples staff to leave but the feedback I got was that the family felt rushed because everybody is getting up and running out of the room ... so now my expectation is that Maples staff stay in the room while I do my piece. I spoke for a little while and then conversations between staff started going on, then I lost the attention of the community members ... it felt chaotic to me. 
International Journal of Child, Youth and Family Studies (2018) 9(1): 54-68

These comments reflect the fact that, as professional practice shifts, it creates new challenges for the entire team. The team's responses must be processed and refined within a collaborative context.

Another important area of practice reflection that emerged was the use of acronyms and diagnostic terminology in the meetings. Terms specific to professional practice were felt to actually create a sense of separation rather than collaboration with families. For example, one community professional identified that "words like 'disregulated' and our knowledge of attachment theory was likely lost on mom". Furthermore, discussions within the CPT generated greater awareness of conveying an unintended meaning when using of some of the terminology common in youth care, and the need to revise our language in this regard. For example, terms frequently used to refer to service delivery such as "residential" and "non-residential" carry significant meaning within Aboriginal communities as they touch on the history of residential schools and the removal of children from families through social services. Accordingly, the organization began the process of finding alternative terminology to use and making the appropriate changes to the program names, descriptions, and manuals.

The topic of when to cancel or reschedule a care plan meeting was another area of practice reflection that arose from the qualitative feedback and discussions within the CPT. For example, in one of the pilot cases, the youth's caregiver was unable to attend the care plan meeting due to a last-minute medical issue. Importantly, this relationship was a strong protective factor in this youth's life after a long history of trauma, loss, and addiction. When asked if she had felt safe in the meeting and if she had felt able to express her opinion regarding who should attend, this youth responded, "If I had had a choice [my caregiver] would have been there." This concern was supported by staff responses, such as, "We should have rescheduled the meeting for this case if we were really paying attention to the TIPs, if we wanted her to feel safe."

Other notes from the in-depth interviews and silent observer data included thoughts for practice reflection such as the need to debrief and check in with each other following a care plan meeting, the related need for peer feedback, and such thoughts as:

Should we offer paper/pen activities to youth in [the] care plan meeting? Understanding why they use music/technology to cope. (CYC) gave [youth] a questionnaire to fill out during the psychology report. Just as it was helpful for mom to read along or take notes, it appeared helpful to have [youth] distract herself with the questionnaire during more difficult times of psych report.

Finally, when asked if this new model had changed or impacted their workload, the team did acknowledge consistently in their feedback that working from a deeper level of connection and trauma awareness actually requires more work in terms of preparing for the meeting, determining what themes to present, deciding how to do so in a trauma-sensitive way, and being present and available to the family during the meeting itself. Comments included: "Maybe over time it will become more efficient as I get used to it. But being trauma-informed will always require more 
International Journal of Child, Youth and Family Studies (2018) 9(1): 54-68

awareness"; "Ya, taking longer prep time, more thinking and awareness and mental prep"; and, "It's harder from our perspective because we are working from a deeper level of awareness". Similarly, an increase in workload was also recognized by the role of the care plan consultant with regard to families "feeling more connected to me so I am fielding calls immediately" and "the role of supporting the care plan in the community starts earlier (now)".

Notwithstanding the identified areas to improve upon, the feedback from this qualitative review also revealed compelling agreement amongst the Maples pilot team that this revised practice was "better" than the previous model. With comments like: "Yes!" and "Absolutely", $100 \%$ of the Maples team agreed that they would recommend this new format to their colleagues.

\section{Conclusion}

In alignment with BC's Trauma-Informed Practice Guide, the qualitative feedback from this review has substantiated the shift in practice of the care plan development meeting as effective in incorporating the principles of a trauma-informed approach. The feedback gathered from the indepth interviews and observational data confirm that this new model was successful in cultivating an environment of safety, choice, and collaboration for clients, and in focusing on the existing strengths of the youth and family, as well as enhancing trauma awareness amongst community stakeholders, the Maples professionals, and the youths and their families.

Furthermore, creating a formalized process that followed a model of qualitative research resulted in the development of clear strategies to shift practice in measurable ways that align with best practices in the field of child and youth mental health. This has resulted in the development of a shift in service delivery that is not only trauma-informed, but is also evidence-based with regard to its efficacy.

As many opportunities for further development have emerged, the CPT is now in the process of in-depth practice reflection pertaining to each area of discipline; templates for reports as well as practice guidelines are now in the early stages of discussion and brainstorming. At the same time, training needs are being identified and plans have been developed to integrate this change into broader practice throughout the organization.

\section{Acknowledgement}

This project is attributable to the ongoing collaborative partnership between Simon Fraser University and the Maples Adolescent Treatment Centre. Furthermore, this research would not have been possible without the participation of the youths, the families, and the community stakeholders involved in each of the pilot cases. We heartily thank them all for their contributions and cooperation. 
International Journal of Child, Youth and Family Studies (2018) 9(1): 54-68

\section{References}

Afifi, T. O., MacMillan, H. L., Boyle, M., Taillieu, T., Cheung, K., \& Sareen, J. (2014). Child abuse and mental disorders in Canada. Canadian Medical Association Journal, 186(9), E324-E332. doi:10.1503/cmaj.131792

Anda, R. F., Felitti, V. J., Bremner, J. D., Walker, J. D., Whitfield, C., Perry, B. D., Dube, S. R., Giles, W. H. (2006). The enduring effects of abuse and related adverse experiences in childhood: A convergence of evidence from neurobiology and epidemiology. European Archives of Psychiatry \& Clinical Neuroscience, 256(3), 174-186. doi:10.1007/s00406005-0624-4

BC Ministry of Children and Family Development. (n.d.). Maples Adolescent Treatment Centre (webpage). Retrieved from https://www2.gov.bc.ca/gov/content/health/managing-yourhealth/mental-health-substance-use/child-teen-mental-health/maples-adolescent-treatmentcentre

BC Provincial Mental Health and Substance Use Planning Council (BCMHSUPC). (2013). Trauma-Informed Practice Guide. Vancouver, BC: Author. Retrieved from http://bccewh.bc.ca/wp-content/uploads/2012/05/2013_TIP-Guide.pdf

BC Provincial Mental Health and Substance Use Planning Council. (2013). Trauma Informed Practice Guide: Key Points.

Bergold, J., \& Thomas, S. (2012). Participatory research methods: A methodological approach in motion. Forum Qualitative Sozialforschung/Forum: Qualitative Social Research, 13(1), Art. 30. doi:10.17169/fqs-13.1.1801

Bouma, G., Ling, R., \& Wilkinson, L. (2012). The research process. Don Mills, ON: Oxford University Press.

Maples Adolescent Treatment Centre. (2014). Operational and Strategic Directional Plan for 2014-2017. Victoria, BC: Ministry of Children and Family Development. 